\title{
A within-person, between-knee comparison of intra- articular platelet-rich plasma versus placebo injection in knees osteoarthritis: a randomized, double-blind comparison
}

\author{
Alireza Sadeghi \\ Zanjan University of Medical Sciences \\ Abdolreza Esmaeilzadeh \\ Zanjan University of Medical Sciences \\ Tayyebeh Lakzaei ( $\sim$ Dr.lakzaei55@zums.ac.ir) \\ Zanjan University of Medical Sciences \\ Saeideh Mazloomzadeh \\ Zanjan University of Medical Sciences \\ Arezoo Karimimoghaddam \\ Zanjan University of Medical Sciences \\ Ali Ammari
}

Zanjan University of Medical Sciences

\section{Research}

Keywords: Osteoarthritis, Intra-Articular Injection, Platelet-Rich Plasma (PRP), Placebo, VAS score, WOMAC.

Posted Date: May 4th, 2021

DOI: https://doi.org/10.21203/rs.3.rs-460265/v1

License: (c) (1) This work is licensed under a Creative Commons Attribution 4.0 International License. Read Full License 


\section{Abstract}

Background Evidence is growing for the efficacy of platelet-rich plasma (PRP) in knee osteoarthritis (OA). Here, we investigated the effects of PRP and placebo by performing a randomized, double-blind, withinperson between knees comparison of measurements.

Methods Thirty patients with equal radiographic grades of OA in both knees received a single injection of PRP in one knee and normal saline as a placebo in the contralateral knee. Knee symptoms were assessed at baseline and at 6-week follow-up using the WOMAC (Western Ontario and McMaster Universities) Osteoarthritis Indices for pain, stiffness, and physical function and the pain visual analog scale (VAS) assessments.

Results The VAS-pain and the mean WOMAC subscales- pain, stiffness, and function- decreased significantly $(P=0.001,0.005,0.024$, and 0.005 , respectively) from baseline to week 6 in the PRP-injected knees, while in the placebo-injected knees there were only a significant reduction of VAS and no difference in WOMAC outcomes. Intergroup comparison of pain-VAS and WOMAC sub-scales revealed no statistically significant differences between PRP- and placebo-treated knees of patients post-injection.

Conclusion This study found little difference between the effects of intra-articular PRP injection and placebo when applied to the same patient. Non-significant improvement in osteoarthritis indices between the treatment and placebo-received knees indicate the need for additional clinical trials of crossover design.

Trial registration: IRCT2016030626922N1

\section{Introduction}

Osteoarthritis (OA) is a chronic debilitating, degenerative disease of the articular cartilage that can be classified into primary and secondary OA $(1,2)$. Primary OA is considered as OA without a clear cause. $O A$ is the most prevalent form of arthritis in the world (3). There is experimental evidence for positive effects of Platelet-Rich Plasma (PRP) in the context of soft tissue healing, ligament and bone regeneration, and inflammation reduction $(4,5)$. Secondary osteoarthritis has a well-known etiology, including endocrine system disorder, anatomical structure abnormality, post-traumatic arthritis, and inflammatory arthritis (6). OA is characterized by the reduction of articular chondrocytes and the destruction of the joint matrix (7). OA is a leading cause of disability that imposes a huge burden to affected individuals, healthcare, and social services worldwide (8). Continuous chondrocyte cartilage damage (9) and articular chondrocyte loss (10) are considered as direct effects of OA. The current available treatment options for knee $\mathrm{OA}$ are few and of limited efficacy. There are some conventional therapies for OA, including physiotherapy, non-steroidal anti-inflammatory drugs, pain-relieving drugs, hyaluronic acid, PRP, or corticosteroid-based intra-articular injections, traditional Chinese medicine, knee arthroscopic surgery and making change of lifestyle like weight loss $(11,12)$. Cartilages are the most important lubricants and shock absorbers between two bones in a joint, the matrix of which contains 
three categories of cells that none of them can reform damaged cartilage as fast as its destruction, so it can end in $O A$ in that joint $(13,14)$. The knee joint is one of the most prevalent affected joints in OA which will finally lead to serious disabilities at the age of more than 65 (15). Platelets are the first cells that arrive in damaged tissues. They contain growth factors that participate in recruiting stem cells and reforming elements to correct the damage (16). PRP consists of a high concentration of growth factors (17). It can boost the healing process by providing the highest concentration of growth factors. One of the most important advantages of PRP is that it is a kind of autologous transplantation, so it has no induced adverse immune reaction $(18,19)$. There are lots of clinical trials and review studies about the improved clinical outcomes acquired from the injection of PRP in the knee joint, but it is not clear if it has a significant effect on the knee with OA repairing or not, yet $(20,21)$. This randomized, double-blind clinical trial was aimed to assess the efficacy of the PRP injection in the treatment of osteoarthritic knees. This research is the first one, to our knowledge, which delineates the clinical outcomes after PRP therapy in patients with bilateral knee osteoarthritis who received PRP treatment in one knee and placebo in the other knee.

\section{Materials And Methods}

This is a randomized double-blind clinical trial study which was conducted in Vali-e-Asr universityaffiliated hospital, Zanjan University of Medical Sciences, Iran. Participants were selected from patients who referred to the rheumatology clinic. Because of the interpersonal differences, we decided to compare patients with themselves. Since both knees of the selected patients were in the same radiographic grade, into one of the knees, PRP was injected (case knee) and the placebo was injected into the other knee (control knee). This study accessed ethic's approval from the Zanjan University of Medical Sciences Research Ethics Committee (number: IRCT2016030626922N1).

\section{Participants}

Knee OA staging was performed by the use of the radiologic Kellgren-Lawrence scale. Our including criteria involved: age of 30 to 70 years old, established diagnosis of knee OA according to ACR criteria, bilateral knee OA with the same Kellgran-Lawrence grade II or III in each knee.

The excluding criteria involved: knee OA due to secondary inflammatory causes, generalized OA, OA with a grade of I or IV, metabolic bone diseases, concomitant back pain, history of intra-articular injection or arthroscopy in previous past 3 months, any kind of severe knee deformity, genu varus or valgus more than 5 degrees, bone tumor, crystal arthropathy, anticoagulant agents use, hemoglobin level less than 10 $\mathrm{mg} / \mathrm{dl}$, platelet dysfunction or count less than 150000, pregnancy, age more than 70 , usage of NSAIDs in last 48 hours, and underlying systemic diseases. Then, by giving them explanations about biosafety, oral consent was reached, and then, written informed consent was achieved.

\section{Interventions}

For producing PRP, participants were pleased to come to the clinic. $50 \mathrm{cc}$ of blood samples were withdrawn by sterile gauge needles and collected into vacutainer blood collection tubes containing 
sodium heparin. We used Beasat Industrial Complex System, Tehran, Iran for PRP preparation.

Then, samples were centrifuged at $1200 \mathrm{rpm}$ for 15 minutes. Centrifuged samples were placed in the extractor for extracting high concentrate platelet plasma. Then the extraction was placed in the centrifuge and was rotated for 6 minutes at $2700 \mathrm{rpm}$. The precipitated component was extracted called PRP. All procedures were conducted in a completely sterilized room of the laboratory. In the end, the concentration of platelets was 4-6 folds of the patients' plasma platelet concentration. 3-5 cc of PRP was injected in knee joint space via supra-patellar root in one knee, and 3-5 cc of normal saline serum (as placebo) was injected in another knee, randomly. All steps were accomplished in a sterile way. At the beginning evaluation and after 6 weeks after the injection, WOMAC and VAS questionnaires were filled up by patients' explanations and the researchers' help. As we chose case and control knees in one person, so we could reduce technical bias as much as possible.

\section{Randomization}

The selection process of the knees for injecting the PRP or placebo was based on the coin random selection method.

\section{Blinding}

Researchers and patients were blinded. Only the rheumatologist who injected PRP and placebo was informed about the type of injection due to separate storage. The syringe containing the placebo and the syringe containing the PRP was covered by a same-colored paper, so that the patient and the researchers did not know about the type of the injections they were received and were given, respectively. Thereafter, these attitudes made our study double-blind.

\section{Primary outcomes}

The primary outcome was analyzed and evaluated through the data received from patients through the WOMAC questionnaire and VAS scores at the beginning of the study. The secondary outcome was the improvement in WOMAC questionnaires and VAS scores 6 weeks after injection. The sample size was 30 patients (60 knees).

\section{Statistical analysis}

All statistical analyses were performed using the Statistical Package for the Social Sciences (SPSS) PC version 16.0 computer software program for Windows (SPSS Inc, Chicago, IL, USA) for acquired data. The Kolmogorov-Smirnov test was used to evaluate the distribution of quantitative variables. Values were expressed as numbers (percentages), and Mean plus-minus Standard Deviation (Mean \pm SD), as appropriate. Comparisons were performed by Paired T-Test for normally distributed, and Wilcox on test for non-normally distributed. Statistical significance was considered at $p$ value $<0.05$.

\section{Results}


Thirty patients (60 knees) were enrolled in the study, 3 were males and 27 were females. Table 1 shows the demographic characteristics of the patients (body mass index, gender, OA radiographic grade ...). In Table 2 and 3, the mean values of WOMAC and VAS scores were compared separately in the knees injected with normal saline and platelet-rich plasma at baseline and after a 6-week follow-up postinjection. The mean score of pain, in PRP injected knees at baseline evaluation and 6 weeks after the injection were 254.83 and 212.00 , respectively $(P=0.005)$. The mean score of joint stiffness in the PRP injected group at baseline was 79.66 and following 6-week follow-up post-injection was $64.16(P=0.024)$. The mean score of joint function in the PRP injected group at baseline was 799.17 and 6 weeks after injection was $668.67(P=0.005)$. Interestingly, the total score of WOMAC in the PRP injected group was also decreased significantly between baseline evaluation and 6 weeks after the first injection $(P=0.005)$. The mean score of VAS in the PRP injected group at baseline evaluation was 63.23 and 6 weeks after the first injection was $50.56(P=0.001)$.

However, for those knees injected with normal saline, only the mean score of VAS decreased significantly $(P=0.012)$. No difference for the mean values of pain, stiffness, function, VAS, and the total score of WOMAC was observed between the knees injected with PRP and those injected with normal saline, at baseline or 6 weeks after the first injection.

In Table 4, the mean difference of WOMAC and VAS scores between baseline evaluation and 6 Weeks after the first injection in PRP and placebo recipient groups, were compared. The mean difference of joint function scores between baseline and 6 weeks after the first injection in PRP injected knees and placebo injected knees was 130.5 and 51.46 , respectively $(P=0.042$, Table 4$)$.

Table 1

Demographic data of the study population $(n=30)$

\begin{tabular}{|llll|}
\hline Variables & Mean \pm SD & Min & Max \\
\hline Weight & $77.166 \pm 9.814$ & 60 & 96 \\
\hline Height & $161.87 \pm 7.57$ & 148 & 182 \\
\hline BMI & $29.53 \pm 4.01$ & 22.58 & 37.11 \\
\hline Gender & Female: $\mathrm{n}=27(90 \%)$ & & \\
& Male: $\mathrm{n}=3(10 \%)$ & & \\
OA grade & Grade $3: \mathrm{n}=21(70 \%)$ & & \\
& Grade $2: \mathrm{n}=9(30 \%)$ & \\
\hline
\end{tabular}

SD: standard deviation, Min: Minimum, Max: Maximum, BMI: Body mass index, OA: Osteoarthritis 
Table 2

Pre- and post-injection comparison of the mean values of VAS scale scoring and WOMAC index for each of the pain, stiffness, and physical function subscores in placebo-treated knees

\begin{tabular}{|llll|}
\hline Variable & $\begin{array}{l}\text { Pre-treatment } \\
(\mathbf{n}=30) \\
\text { Mean } \pm \text { SD }\end{array}$ & $\begin{array}{l}\text { Post-treatment } \\
(\mathbf{n}=\mathbf{3 0})\end{array}$ & P value \\
\hline Pain & $234.5 \pm 130.18$ & $211.32 \pm 133.99$ & 0.163 \\
\hline Stiffness & $75.50 \pm 60.66$ & $66.66 \pm 53.18$ & 0.197 \\
\hline Physical function & $726.17 \pm 411.47$ & $674.70 \pm 427.46$ & 0.333 \\
\hline Total WOMAC score & $1036.2 \pm 563.64$ & $952.68 \pm 584.72$ & 0.262 \\
\hline VAS score & $59.23 \pm 27.79$ & $50.06 \pm 29.32$ & 0.012 \\
\hline
\end{tabular}

WOMAC: The Western Ontario and McMaster Universities Osteoarthritis Index, VAS: Visual Analog Scale, PRP: Platelet-Rich Plasma, SD: Standard deviation.

Table 3

Pre- and post-injection comparison of the mean values of VAS scale scoring and WOMAC index for each of the pain, stiffness, and physical function subscores in PRP-injected knees

\begin{tabular}{|llll|}
\hline Variable & $\begin{array}{l}\text { Pre-treatment } \\
(\mathbf{n}=30) \\
\text { Mean } \pm \text { SD }\end{array}$ & $\begin{array}{l}\text { Post-treatment } \\
(\mathbf{n}=\mathbf{3 0}) \\
\text { Mean } \pm \text { SD }\end{array}$ & P value \\
\hline Pain & $254.83 \pm 118.86$ & $212.00 \pm 128.2$ & 0.005 \\
\hline Stiffness & $79.66 \pm 58.57$ & $64.16 \pm 54.93$ & 0.024 \\
\hline Physical function & $799.17 \pm 379.280$ & $668.67 \pm 376.05$ & 0.005 \\
\hline Total WOMAC score & $1133.7 \pm 520.80$ & $944.8 \pm 531.96$ & 0.005 \\
\hline VAS score & $63.23 \pm 26.40$ & $50.56 \pm 28.04$ & 0.001 \\
\hline
\end{tabular}

WOMAC: The Western Ontario and McMaster Universities Osteoarthritis Index, VAS: Visual Analog Scale, PRP: Platelet-Rich Plasma, SD: Standard deviation. 
Table 4

Intergroup comparison of two groups for VAS score and WOMAC subscores at baseline

\begin{tabular}{|llll|}
\hline Variable & $\begin{array}{l}\text { PRP-injected knees } \\
(\mathbf{n = 3 0}) \\
\text { Mean } \pm \text { SD }\end{array}$ & $\begin{array}{l}\text { Placebo-injected knees } \\
(\mathbf{n}=\mathbf{3 0}) \\
\text { Mean } \pm \text { SD }\end{array}$ & P value \\
\hline Pain & $254.83 \pm 118.86$ & $234.5 \pm 130.18$ & 0.180 \\
\hline Stiffness & $79.66 \pm 58.57$ & $75.5 \pm 60.66$ & 0.548 \\
\hline Physical function & $799.17 \pm 379.28$ & $726.17 \pm 411.47$ & 0.09 \\
\hline Total WOMAC & $1133.7 \pm 520.80$ & $1036.2 \pm 563.64$ & 0.118 \\
score & & & 0.165 \\
\hline VAS score & $63.23 \pm 26.40$ & $59.23 \pm 27.79$ & \\
\hline
\end{tabular}

WOMAC: The Western Ontario and McMaster Universities Osteoarthritis Index, VAS: Visual Analog Scale, PRP: Platelet-Rich Plasma, SD: Standard deviation.

Table 5

Intergroup comparison of two groups for VAS score and WOMAC sub-scores post treatment

\begin{tabular}{|llll|}
\hline Variable & $\begin{array}{l}\text { PRP-injected knees } \\
(\mathbf{n}=\mathbf{3 0}) \\
\text { Mean } \pm \text { SD }\end{array}$ & $\begin{array}{l}\text { Saline-injected knees } \\
(\mathbf{n}=\mathbf{3 0}) \\
\text { Mean } \pm \text { SD }\end{array}$ & P value \\
\hline Pain & $212.22 \pm 128.20$ & $211.32 \pm 133.99$ & 0.966 \\
\hline Stiffness & $64.16 \pm 54.93$ & $66.66 \pm 53.18$ & 0.723 \\
\hline Physical function & $668.67 \pm 376.05$ & $674.7 \pm 427.46$ & 0.09 \\
\hline Total WOMAC & $944.83 \pm 531.96$ & $952.68 \pm 584.72$ & 0.904 \\
score & & & 0.869 \\
\hline VAS score & $50.56 \pm 28.04$ & $50.06 \pm 29.32$ & \\
\hline
\end{tabular}


WOMAC: The Western Ontario and McMaster Universities Osteoarthritis Index, VAS: Visual Analog Scale, PRP: Platelet-Rich Plasma, SD: Standard deviation.

\section{Discussion}

In light of the growing evidence of its benefit in orthopedics, we sought to explore the effects of PRP injection on adults with osteoarthritis of the knee. The use of PRP injection in patients affected by knee osteoarthritis has been extensively studied, but clinical outcomes are still highly variable (21-23). This analysis was carried out taking into account the current variations in treatment outcomes of patients with OA, noted in prior studies. The lack of standardization among the protocols used for PRP therapy may have contributed to the variability of most of the results (24). In addition, there is a large interpersonal variation in therapeutic response to this biologic treatment approach in OA patients (25). This could be due to a variety of causes including the influence of the individual patients' characteristics, comorbidities, and concurrent medications $(26,27)$. Several placebo-controlled trials have carried out on the PRP of patients with knee $O A$ and provided conflicting evidence regarding their efficacy $(21,28-30)$. The aim of this study was to compare the efficacy and efficiency of PRP versus placebo in patients with bilateral knee OA by a blinded comparison to see how the treatment compares to the placebo for the treatment of knee OA. Comparing physical function, stiffness and the degree of pain in both knees of the same patient can reduce the individual variance in the perception of outcome parameters. We thus applied a withinperson, between-knee study in subjects with the same radiographic OA grade 3 or 2 in both knees. We specifically excluded grade 1 and 4 OA patients to ensure sufficient homogeneity of the study population and methodology. This is an important consideration, because treatment with PRP is not routinely performed in severe OA (Kellgren Lawrence grade 4) who has concurrent bone deformity (31). In our study, patients who had different KL grades on the two sides at baseline were excluded, to provide a homogeneous group. In addition, a double-blind protocol was employed in administering the drug and a saline control to minimize bias in comparing the results. Since, according to a recent work, responsiveness to PRP are expected to be influenced by the receiver's features (32), it was suggested that finding the true scientific evidence for PRP in the treatment of patients with OA requires a strong study design to properly estimate the benefits of PRP. On the other hand, one significant issue is the large impact of placebo in the setting of intra-articular injections (33), which makes it difficult to confirm the therapeutic potential of PRP in managing the knee OA. In an RCT in 2013, 78 patients with knee OA were randomized to receive PRP or saline. In that trial, the effectiveness of PRP was investigated by using a placebo-controlled study design (34); one group received PRP, and a separate control group received saline injections. In the present study, however, we included only patients with bilateral knee OA and both knees of the same patient were injected individually with PRP or saline. The originality of the current study is that the therapeutic schema was evaluated on a case-by-case approach and values were calculated separately for each patient. In our study, comparison between pre and post intervention values of WOMAC pain, stiffness and physical function showed significant difference in PRP-injected knees but saline-injected knees did not yield any significant results. Surprisingly, knees injected with saline showed 
evidence of decreased visual pain analog scores $(p=0.012)$. Furthermore, a statistically non-significant, but clinically relevant improvement of the stiffness and WOMAC pain was also found in the knees injected with saline. When PRP injection was compared to placebo, the results of the PRP were not statistically significant in the WOMAC sub-scores and VAS score, however, there was significant change in the outcome variables of PRP injection from baseline to 6-week follow-up. With respect to the observed non-significant difference between PRP and placebo effects, our finding is consistent with a previous systematic review and meta-analysis that found that the clinical outcomes in terms of WOMAC pain, stiffness, and physical function is not significantly better in patients undergoing PRP for OA when compared to those in patients treated with hyaluronic acid or placebo (35). However, the study indicates that PRP injection is an effective treatment for OA of the knee with beneficial effects on WOMAC total score, IKDC score and EQ-VAS score compared to hyaluronic acid injection (35).

From this observation, it can be hypothesized that PRP administration not only has local effects in OA joints but also could exert systemic reactions in our study. This hypothesis may explain the pain relief in short-term following PRP administration observed in the placebo treated group and may account for statistically significant VAS score reduction linked to the clinical placebo effects. Mechanisms involved in the pathophysiology of OA include a combination of mechanical, cellular and biochemical changes which damage on mechanical properties of the articular cartilage (36). The clinical progress of OA is influenced by an imbalance between proinflammatory and anti-inflammatory mediators which promotes a strong inflammatory reaction and thereby activates proteolytic enzymes that destroy cartilage (37). Based on the findings of experimental studies, (38) and clinical examinations $(39,40)$, the mechanism of bilateral effect of unilateral PRP injection is presumably due to potential systemic effects after localized PRP injection that suggests local administration of PRP may affect the outcome not only for the ipsilateral knee but also for the contralateral side untreated with PRP. This is consistent with a recently published report by Kou et al. that showed a significant decrease in the serum substance P (SP) level after local PRP injection in sports injuries, which then led to decreased nociception secondary to the healing process after PRP injection (41). Second, and more importantly, evidence for a positive correlation between the serum SP concentration and chronic pain in patients with OA also has been reported by Lisowska et al. (2015) (42). From reviewing the literature, a number of conclusions can be drawn. A recent systematic review of literature and meta-analysis evaluating intra-articular PRP injections in patients with knee OA revealed that PRP injections do not induce direct cartilage regeneration but rather works through a reduction of inflammatory distress that result in modulation of articular cartilage degradation (32). This presents the biological rationale linking local PRP injection to systemic antiinflammatory effects, reported for this intervention. Notably, for a better understanding of the bilateral effect of unilateral PRP, further investigations may have been required. In this regard, future studies will look at how the local PRP injection compares to no treatment at all, at the other side, which may be called a baseline condition to verify whether the effects of local PRP therapy are able to improve the contralateral knee symptoms.

Our study has some obvious limitations, e.g. the number of subjects studied was small and follow-up time was limited. A larger study need to continue the current study design with follow-up periods over a 
longer period of time to expand the current findings. Second, we did not measure levels of cytokines or growth factors to test possible mechanisms linking locally injected PRP to secondary systemic effects which could provide useful information on the bioactivity of PRP. To conclude, this study was developed for monitoring the efficacy of PRP therapy under controlled conditions, via examining clinical outcomes in individual patients who received concurrent PRP versus placebo that allows finding out the actual response of an individual patient to PRP administration. With comparison of each subject's baseline score with the subject's own treatment phase scores for both treatments, the effects of confounding factors, such as age, gender, body mass Index (BMI) on osteoarthritis indices was minimized. Perhaps most importantly, our results indicate that if a systemic effect is really present, intra-articular application of PRP can have beneficial effects on overall care of patients who has primary bilateral osteoarthritis of the knee. It is important to try to determine the extent of the placebo effects, and how they contribute to the specific effects of therapy.

\section{Declarations}

\section{Ethics approval and consent to participate}

Informed consent was obtained from all subjects involved in the study. The study was conducted according to the guidelines of the Declaration of Helsinki, and approved by the Institutional Review Board (or Ethics Committee) of Zanjan University of Medical Sciences Research Ethics Committee (IRCT2016030626922N1).

\section{Consent for publication}

Not applicable.

\section{Availability of data and materials}

The data presented in this study are available on request from the corresponding author.

\section{Competing interests}

The authors declare that they have no competing interests.

\section{Funding}

This research was funded by Zanjan University of Medical Sciences, Zanjan, Iran grant number A-12-6025 .

\section{Author Contributions}

Conceptualization, A.S. and A.E.; Methodology, T.L.; Writing - Original Draft Preparation, T.L.; Data Curation, S.M. and A.K.; Supervision, A.S. and A.E.; Project Administration, A.S.; Writing - Review \& Editing, A.A.. 
Acknowledgements

Not applicable.

\section{References}

1. Abramson SB, Attur M. Developments in the scientific understanding of osteoarthritis. Arthritis research \& therapy. 2009;11(3):227.

2. Tchetina EV. Developmental mechanisms in articular cartilage degradation in osteoarthritis. Arthritis. 2011;2011.

3. Bijlsma JW, Berenbaum F, Lafeber FP. Osteoarthritis: an update with relevance for clinical practice. The Lancet. 2011;377(9783):2115-26.

4. Steinert AF, Middleton KK, Araujo PH, Fu FH. Platelet-rich plasma in orthopaedic surgery and sports medicine: pearls, pitfalls, and new trends in research. Operative Techniques in Orthopaedics. 2012;22(2):91-103.

5. Rodriguez-Merchan EC. Intraarticular injections of platelet-rich plasma (PRP) in the management of knee osteoarthritis. Archives of Bone and Joint Surgery. 2013;1(1):5.

6. Koh Y-G, Choi Y-J. Infrapatellar fat pad-derived mesenchymal stem cell therapy for knee osteoarthritis. The Knee. 2012;19(6):902-7.

7. Ghosh P, Smith M. Osteoarthritis, genetic and molecular mechanisms. Biogerontology. 2002;3(12):85-8.

8. Vos T, Flaxman AD, Naghavi M, Lozano R, Michaud C, Ezzati M, et al. Years lived with disability (YLDs) for 1160 sequelae of 289 diseases and injuries 1990-2010: a systematic analysis for the Global Burden of Disease Study 2010. The lancet. 2012;380(9859):2163-96.

9. Vega A, Martín-Ferrero MA, Del Canto F, Alberca M, García V, Munar A, et al. Treatment of knee osteoarthritis with allogeneic bone marrow mesenchymal stem cells: a randomized controlled trial. Transplantation. 2015;99(8):1681-90.

10. Pers Y-M, Rackwitz L, Ferreira R, Pullig O, Delfour C, Barry F, et al. Adipose mesenchymal stromal cellbased therapy for severe osteoarthritis of the knee: A phase i dose-escalation trial. Stem cells translational medicine. 2016;5(7):847-56.

11. Freitag J, Bates D, Boyd R, Shah K, Barnard A, Huguenin L, et al. Mesenchymal stem cell therapy in the treatment of osteoarthritis: reparative pathways, safety and efficacy-a review. BMC musculoskeletal disorders. 2016;17(1):230.

12. Sadeghi A, Rad ZA, Sajedi B, Heydari AH, Akbarieh S, Jafari B. Effect of weight losing on the clinical status improvement of patients with knee osteoarthritis. Reumatologia clinica. 2019;15(2):73-6.

13. Pap T, Korb-Pap A. Cartilage damage in osteoarthritis and rheumatoid arthritis-two unequal siblings. Nature Reviews Rheumatology. 2015;11(10):606-15. 
14. Song Yz, Guan J, Wang Hj, Ma W, Li F, Xu F, et al. Possible involvement of serum and synovial fluid resistin in knee osteoarthritis: cartilage damage, clinical, and radiological links. Journal of clinical laboratory analysis. 2016;30(5):437-43.

15. Vina E, Ran D, Ashbeck E, Kwoh C. AB0754 Racial Differences in Knee Osteoarthritis Pain, Symptoms and Disability Reporting. BMJ Publishing Group Ltd; 2016.

16. Cancedda R, Bollini S, Descalzi F, Mastrogiacomo M, Tasso R. Learning from mother nature: innovative tools to boost endogenous repair of critical or difficult-to-heal large tissue defects. Frontiers in bioengineering and biotechnology. 2017;5:28.

17. Peerbooms JC, Sluimer J, Bruijn DJ, Gosens T. Positive effect of an autologous platelet concentrate in lateral epicondylitis in a double-blind randomized controlled trial: platelet-rich plasma versus corticosteroid injection with a 1-year follow-up. The American journal of sports medicine. 2010;38(2):255-62.

18. Padilla S, Anitua E, Fiz N, Pompei O, Azofra J, Sánchez M. The scientific rationale to apply plasma rich in growth factors in joint tissue pathologies: knee osteoarthritis. Platelet Rich Plasma in Orthopaedics and Sports Medicine: Springer; 2018. p. 125-43.

19. Gobbi A, Lad D, Karnatzikos G. The effects of repeated intra-articular PRP injections on clinical outcomes of early osteoarthritis of the knee. Knee Surgery, Sports Traumatology, Arthroscopy. 2015;23(8):2170-7.

20. Raeissadat SA, Rayegani SM, Hassanabadi H, Fathi M, Ghorbani E, Babaee M, et al. Knee osteoarthritis injection choices: platelet-rich plasma (PRP) versus hyaluronic acid (a one-year randomized clinical trial). Clinical Medicine Insights: Arthritis and Musculoskeletal Disorders. 2015;8:CMAMD. S17894.

21. Görmeli G, Görmeli CA, Ataoglu B, Çolak C, Aslantürk O, Ertem K. Multiple PRP injections are more effective than single injections and hyaluronic acid in knees with early osteoarthritis: a randomized, double-blind, placebo-controlled trial. Knee Surgery, Sports Traumatology, Arthroscopy. 2017;25(3):958-65.

22. Guillibert C, Charpin C, Raffray M, Benmenni A, Dehaut F-X, El Ghobeira G, et al. Single injection of high volume of autologous pure PRP provides a significant improvement in knee osteoarthritis: a prospective routine care study. International journal of molecular sciences. 2019;20(6):1327.

23. Jang S-J, Kim J-D, Cha S-S. Platelet-rich plasma (PRP) injections as an effective treatment for early osteoarthritis. European Journal of Orthopaedic Surgery \& Traumatology. 2013;23(5):573-80.

24. Milants $C$, Bruyère 0, Kaux J-F. Responders to platelet-rich plasma in osteoarthritis: a technical analysis. BioMed Research International. 2017;2017.

25. O’Donnell C, Migliore E, Grandi FC, Koltsov J, Lingampalli N, Cisar C, et al. Platelet-Rich Plasma (PRP) From Older Males With Knee Osteoarthritis Depresses Chondrocyte Metabolism and Upregulates Inflammation. Journal of Orthopaedic Research®. 2019;37(8):1760-70.

26. Di Matteo B, Filardo G, Lo Presti M, Kon E, Marcacci M. Chronic anti-platelet therapy: a contraindication for platelet-rich plasma intra-articular injections. Eur Rev Med Pharmacol Sci. 
2014;18(1 Suppl):55-9.

27. Velier M, Magalon J, Daumas A, Cassar M, Francois P, Ghazouane A, et al. Production of platelet-rich plasma gel from elderly patients under antithrombotic drugs: perspectives in chronic wounds care. Platelets. 2018;29(5):496-503.

28. Huang G, Hua S, Yang T, Ma J, Yu W, Chen X. Platelet-rich plasma shows beneficial effects for patients with knee osteoarthritis by suppressing inflammatory factors. Experimental and therapeutic medicine. 2018;15(3):3096-102.

29. Krogh TP, Ellingsen T, Christensen R, Jensen P, Fredberg U. Ultrasound-guided injection therapy of Achilles tendinopathy with platelet-rich plasma or saline: a randomized, blinded, placebo-controlled trial. The American journal of sports medicine. 2016;44(8):1990-7.

30. Lin K-Y, Yang C-C, Hsu C-J, Yeh M-L, Renn J-H. Intra-articular injection of platelet-rich plasma is superior to hyaluronic acid or saline solution in the treatment of mild to moderate knee osteoarthritis: a randomized, double-blind, triple-parallel, placebo-controlled clinical trial. Arthroscopy: The Journal of Arthroscopic \& Related Surgery. 2019;35(1):106-17.

31. Bendich I, Rubenstein WJ, Cole BJ, Ma CB, Feeley BT, Lansdown DA. What Is the Appropriate Price for Platelet-Rich Plasma Injections for Knee Osteoarthritis? A Cost-Effectiveness Analysis Based on Evidence From Level I Randomized Controlled Trials. Arthroscopy: The Journal of Arthroscopic \& Related Surgery. 2020.

32. Kon E, Di Matteo B, Delgado D, Cole BJ, Dorotei A, Dragoo JL, et al. Platelet-rich plasma for the treatment of knee osteoarthritis: an expert opinion and proposal for a novel classification and coding system. Expert Opinion on Biological Therapy. 2020:1-14.

33. Previtali D, Merli G, Di Laura Frattura G, Candrian C, Zaffagnini S, Filardo G. The Long-Lasting Effects of "Placebo Injections" in Knee Osteoarthritis: A Meta-Analysis. Cartilage. 2020:1947603520906597.

34. Patel S, Dhillon MS, Aggarwal S, Marwaha N, Jain A. Treatment with platelet-rich plasma is more effective than placebo for knee osteoarthritis: a prospective, double-blind, randomized trial. The American journal of sports medicine. 2013;41(2):356-64.

35. Kanchanatawan W, Arirachakaran A, Chaijenkij K, Prasathaporn N, Boonard M, Piyapittayanun P, et al. Short-term outcomes of platelet-rich plasma injection for treatment of osteoarthritis of the knee. Knee Surgery, Sports Traumatology, Arthroscopy. 2016;24(5):1665-77.

36. Buckwalter JA, Mankin HJ, Grodzinsky AJ. Articular cartilage and osteoarthritis. Instructional Course Lectures-American Academy of Orthopaedic Surgeons. 2005;54:465.

37. Carballo CB, Nakagawa Y, Sekiya I, Rodeo SA. Basic science of articular cartilage. Clinics in sports medicine. 2017;36(3):413-25.

38. Borrione P, Grasso L, Chierto E, Geuna S, Racca S, Abbadessa G, et al. Experimental model for the study of the effects of platelet-rich plasma on the early phases of muscle healing. Blood Transfusion. 2014;12(Suppl 1):s221.

39. Borrione P, Fossati C, Pereira MT, Giannini S, Davico M, Minganti C, et al. The use of platelet-rich plasma (PRP) in the treatment of gastrocnemius strains: a retrospective observational study. 
Platelets. 2018;29(6):596-601.

40. Banfi G, Corsi MM, Volpi P. Could platelet rich plasma have effects on systemic circulating growth factors and cytokine release in orthopaedic applications? : British Association of Sport and Excercise Medicine; 2006.

41. Xie X, Wu H, Zhao S, Xie G, Huangfu X, Zhao J. The effect of platelet-rich plasma on patterns of gene expression in a dog model of anterior cruciate ligament reconstruction. journal of surgical research. 2013;180(1):80-8.

42. Lisowska B, Lisowski A, Siewruk K. Substance $P$ and chronic pain in patients with chronic inflammation of connective tissue. PloS one. 2015;10(10):e0139206. 